OPEN ACCESS

Edited by:

Leon De Bruin,

Radboud University Nijmegen,

Netherlands

Reviewed by:

Fernando Marmolejo-Ramos, University of South Australia, Australia Ophelia Deroy,

Ludwig Maximilian University of Munich, Germany

*Correspondence: Artur Czeszumski aczeszumski@uos.de

Specialty section: This article was submitted to Theoretical and Philosophical

Psychology,

a section of the journal

Frontiers in Psychology

Received: 09 September 2020 Accepted: 18 February 2021

Published: 15 March 2021

Citation:

Czeszumski A, Albers F, Walter $S$ and König P (2021) Let Me Make You

Happy, and I'll Tell You How You Look Around: Using an

Approach-Avoidance Task as an Embodied Emotion Prime in a Free-Viewing Task.

Front. Psychol. 12:604393 doi: 10.3389/fpsyg.2021.604393

\section{Let Me Make You Happy, and I'll Tell You How You Look Around: Using an Approach-Avoidance Task as an Embodied Emotion Prime in a Free-Viewing Task}

\author{
Artur Czeszumski ${ }^{1 *}$, Friederike Albers ${ }^{1}$, Sven Walter $^{1}$ and Peter König ${ }^{1,2}$ \\ ${ }^{1}$ Institute of Cognitive Science, Universität Osnabrück, Osnabrück, Germany, ${ }^{2}$ Institut für Neurophysiologie und \\ Pathophysiologie, Universitätsklinikum Hamburg-Eppendorf, Hamburg, Germany
}

The embodied approach of human cognition suggests that concepts are deeply dependent upon and constrained by an agent's physical body's characteristics, such as performed body movements. In this study, we attempted to broaden previous research on emotional priming, investigating the interaction of emotions and visual exploration. We used the joystick-based approach-avoidance task to influence the emotional states of participants, and subsequently, we presented pictures of news web pages on a computer screen and measured participant's eye movements. As a result, the number of fixations on images increased, the total dwell time increased, and the average saccade length from outside of the images toward the images decreased after the bodily congruent priming phase. The combination of these effects suggests increased attention to web pages' image content after the participants performed bodily congruent actions in the priming phase. Thus, congruent bodily interaction with images in the priming phase fosters visual interaction in the subsequent exploration phase.

\footnotetext{
Keywords: overt attention, eye-tracking, emotions, cognitive bias modification, automatic approach bias, embodiment, approach avoidance task
}

\section{INTRODUCTION}

Gaze-dependent shifts play a pivotal role in visual processing. Using modern eye-tracking techniques, it is possible to measure overt shifts of attention reliably and unobtrusively, helping us understand eye movement behavior. What one observes is influenced by at least three factors. First, attention is influenced by the external stimuli' properties, processed in a bottom-up hierarchy (Treisman and Gelade, 1980; Itti and Koch, 2000). This includes low-level features of the visual stimulus, for instance, contrast, contours, color, texture, and motion. However, it may also include more complex features like complex shapes of objects or the emotional valence of images (Thomas and Hasher, 2006; Einhäuser et al., 2008). Second, attention is influenced by internal variables like task-demands (Hayhoe et al., 2003; Einhäuser and Koch, 2008; Rothkopf et al., 2016), as well as the observer's emotional state (Kaspar et al., 2013). Third, the spatial factors like the central bias (Tatler, 2007) and saccadic momentum (Wilming et al., 2013) influence the selection of fixation targets. These three factors' relative contribution is a matter of debate (Kollmorgen et al., 2010), and presumably depends on the precise circumstances (Einhäuser and Koch, 2008). Additionally, to all 
different mentioned levels that attention can be influenced, it is crucial to operationalize attention itself (Hommel et al., 2019). Our study used direction and allocation of eye movements to refer to attention (Rayner, 2009).

When it comes to the role of emotional states affecting attention, it is useful to distinguish between an internal affective influence, e.g., the emotional state of the observer, and an external affective influence, e.g., the stimulus valence (Damasio, 1999; Kaspar and König, 2012; Kaspar et al., 2013, 2015; Colombetti, 2014). A situation in which attention is subject to both external and internal affective influences is when one explores web pages of online news portals. On the one hand, such web pages commonly contain positive alongside negative information, whereas, on the other hand, one is in a specific emotional state: Positive, negative, or neutral. Kaspar et al. (2015) used such an environment to investigate the internal and external affective influences in a free-viewing task performed by young adults. The participants' emotional state was primed by a series of either positively or negatively valenced visual stimuli. Subsequently, they had to explore web pages containing both positively and negatively valenced content. An analysis of the eye-tracking data revealed that a negative emotional state marginally elicited a more spatially extensive exploration and that attention for negative news increased in participants who were in a positive emotional state. Thus, the state of the observer and the external affective influence impacted the visual exploration.

The valence of the stimulus influences responses beyond visual exploration. Specifically, approach-behavior is naturally associated with an appraisal of something as "good." In contrast, avoidance behavior is naturally associated with an appraisal of something as "bad." As a consequence, we are faster (Chen and Bargh, 1999) and more accurate (Casasanto and Dijkstra, 2010) when making movements that correspond to their embodied meaning, i.e., approach for good, and avoid for bad. In particular, there is a general bodily tendency to approach positive and avoid negative cues and do so faster than vice versa (Phaf et al., 2014). Moreover, it was shown that positive concepts and percepts are placed close-to-the-body locations, while negative concepts and percepts are placed away-from-the-body locations (Marmolejo-Ramos et al., 2018, 2019). This gives evidence for a general bodily reaction to positive or negative stimuli (Phaf et al., 2014; Sharbanee et al., 2014). For example, spider phobics avoid pictures of spiders more strongly than neutral cues (Rinck and Becker, 2007); socially anxious people avoid smiling and angry faces faster than controls (Heuer et al., 2007); schizophrenic patients with higher levels of oxytocin avoid angry faces faster than controls (Brown et al., 2014); individuals with anorexianervosa exhibit a decreased approach bias for food cues (Veenstra and de Jong, 2011); and healthy adults pull positive words faster toward them while pushing negative words faster away (Chen and Bargh, 1999). Thus, the valence of stimuli has a widespread impact on bodily states and actions.

The cognitive mechanisms of the automatic approach bias are still debated. One approach, the concept of embodied cognition, rejects the idea that an agent's cognitive life can be understood without considering the particular morphological, biological, and physiological characteristics of its body (Shapiro,
2011; Engel et al., 2013; Walter, 2014). For instance, language processing (Glenberg and Kaschak, 2002), memory (Casasanto and Dijkstra, 2010), visual-motor recalibration (Bhalla and Proffitt, 1999), or distance estimation (Witt and Proffitt, 2008) all rely on specific body characteristics. Moreover, even our abstract concepts are bodily "grounded" and arise from the body (Barsalou, 2008). That is to say that according to the embodied approach of cognition and affectivity, cognitive and affective phenomena can be fully understood only by taking into account the specific morphological, biological, and physiological details of the agent's body (Shapiro, 2011; Engel et al., 2013; Walter, 2014). In particular, bodily movements are specific to the kind of body we have, and to the environment, we interact with, and are thus naturally meaningful. Similarly, the embodied approach to cognition tries to explain the approach-avoidance behavior (Fridland and Wiers, 2018). Importantly for our study, affective states are also considered within the embodied cognition framework. Stephan et al. (2014) discuss emotions in relation to the body and beyond the body and the brain. Furthermore, Slaby et al. (2016) propose an action-oriented understanding of emotions.

However, although emotional priming has been an important topic in research on top-down influences in overt attention, in particular when it comes to disentangling external and internal affective influences, there is little research using embodied primes (Stoykov et al., 2017). As creatures with specific bodily morphology, our onto- and phylogeny make it natural that positive valence is pulling something toward us while pushing it away is negatively valenced (Fridland and Wiers, 2018). Since the human abdominal region is exceptionally vulnerable, we have to protect it by allowing only trustworthy objects to come close. Since survival requires energy, we have to pull nourishing objects toward us while avoiding rotten, poisonous, unsanitary, or noxious objects. While strangers must typically be kept at bay, procreation, nurturing infants, and giving them love and comfort require social approaching. The idea that approachand avoidance-behavior is naturally associated with appraisals of something as "good" or "bad" is also in line with embodied accounts of emotions (Niedenthal et al., 2005; Stephan et al., 2014), in particular with Damasio's (Damasio, 2001) "somatic marker" theory, according to which emotions function to direct animals toward what is good and direct them away from what is bad. Hence, these considerations suggest that approaching something or pulling it toward us is naturally meaningful, indicating something is positive.

If the automatic approach bias is indeed a general bodily reaction to positive or negative stimuli (Phaf et al., 2014), we should observe it in healthy adults performing an approachavoidance task. This type of explanation raises new questions. Namely, if the bodily relation is crucial, we would expect an influence of the stimulus valence and a congruency effect. Body movements that are in line with our preferences (pull toward positive, "good"/push away negative, "bad") should influence our eye movements differently than priming by incongruent actions preferences (pull toward negative, "bad"/push away positive, "good"). Therefore, we aim to answer that question with our design. The congruency effect would give support to the claim 
that embodied priming modulates our viewing behavior. That is, here, we are primarily interested in modulating the natural (embodied) action in response to a stimulus e.g., congruent vs. incongruent, as well as investigating the effects on subsequent visual exploratory behavior.

The present study builds on an embodied approach to the automatic approach bias in order to investigate (1) whether healthy adults exhibit a comparable automatic approach bias concerning positively and negatively valenced stimuli and (2) how a positive vs. negative emotional state, induced by a congruent vs. incongruent approach-avoidance task affects their overt attention in a free-viewing task.

\section{METHODS}

\section{Participants}

Twenty participants (6 male, 18 right-handed, mean age of 22.6 years, standard deviation of 2 years) took part in the experiment. They gave written informed consent before the start of the experiment. Participants received either $9 €$ or course credits in exchange for their participation. All participants had normal or corrected to normal vision and were not aware of the study's scientific purpose. They were either native German speakers or fluent in the German language. This was important since the presented stimuli included headlines written in German. The ethics committee of Osnabrück University approved the study.

\section{General Apparatus}

We presented all stimuli on a 24" LCD monitor (BenQ XL2420T; BenQ, Taipeh, Taiwan) with a refresh rate of $114 \mathrm{~Hz}$. Participants sat $80 \mathrm{~cm}$ away from the screen. The experiment was controlled by a PC (Dell) connected to an eye tracker computer via an Ethernet cable. We used a head-mounted eye tracker (Eye Link II system) from SR-Research Ltd. (SR-Research Ltd, Ontario, Canada) to track the participants' eye movements. In turn, the eye tracker was connected to a DOS-based computer (Pentium 4; Dell, Round Rock, TX, USA) running the application software. In total, the eye tracker comprised three infrared cameras. The head camera recorded infrared sensors attached to the monitor's corners to calculate the head position in relation to the screen continuously. This allowed a stable gaze recording irrespective of involuntary small head movements. The other two infrared cameras recorded the participants' pupil positions. The sampling rate of binocular recordings was $500 \mathrm{~Hz}$. The room was darkened during the entire experiment.

A 13-point calibration task preceded each recording. It consisted of fixation points appearing consecutively in random order at various screen locations, and participants were instructed to focus their gaze at these points. Each point had a visual angle size of $0.5^{\circ}$. We validated the calibration by calculating the drift error for each point. Thereby it was assured that the mean validation error stayed below a $0.3^{\circ}$ visual angle and the maximum validation error below a $1^{\circ}$ visual angle. The calibration was repeated until the mentioned accuracy was reached.

We used the eye tracker's default settings to calculate saccades and fixations. Saccade detection was based on a velocity of at least $30^{\circ}$ visual angle/s and acceleration of at least $8,000^{\circ}$ visual angles $/ \mathrm{s}^{2}$. To trigger a saccade, the saccade signal had to be sustained for at least $4 \mathrm{~ms}$. By the time the eyes moved significantly from the fixation point (i.e., exceeding a motion threshold), the saccade's temporal and spatial onset had been defined. By default, we set this motion threshold to a $0.1^{\circ}$ visual degree. After the saccade onset, the minimal saccade velocity was $25^{\circ}$ visual degree/s. Following this, a period without a saccade was marked as fixation. Each trial was followed by a fixation cross appearing in the screen center to control drifts in measurements. The first fixation following each stimulus's onset was excluded from our analysis because this was an artifact from the drift correction before the respective trial's onset.

The joystick used for the approach-avoidance task (Logitech Attack TM 3; Logitech, Apples, Switzerland) was connected to the computer screen. Matlab's Psychtoolbox V3 (r2017a; MathWorks Company) enabled us to record response times (pushing/pulling movements). The joystick was placed on the table in front of the participants. We used MATLAB to preprocess eye-tracking data and $\mathrm{R}$ to analyze all data. Analysis scripts and data are available online (https://osf.io/cyz9b/).

\section{Stimuli}

The experiment included two separate phases (see below for details). First, participants performed an approach-avoidance task, viewing isolated images. Afterward, participants visually explored web pages, each containing two embedded images with additional text columns in a typical newspaper layout. As we investigated the influence of the approach-avoidance task on later visual exploration, we labeled the isolated images as "primes."

In this study, we used 88 full-colored images from the International Affective Picture Set (IAPS) (Lang et al., 1997). Kaspar et al. (2015) used the identical stimulus set. Half of the images had a valence rated below 3 (IAPS scale) and served as negative primes. The other 44 images had valence ratings above 7 and served as positive primes. To prevent the images from blurring, we presented all of them in their native resolution of $1,024 \times 768$ pixels on a gray background (RGB values: $182 / 182 / 182$ ), centered in the middle of the screen (resolution of $1,920 \times 1,080$ pixels, Figure $2 \mathrm{~A})$.

In the present study, twenty-four prototypes of news web pages were used, previously designed by Kaspar et al. (2015) (Figure 1). The web page images' resolution fits the screens' resolution $(1,920 \times 1,080)$. Two target areas, embedded by several textual and pictorial components, were constructed in one web page design (Figure 1A). Each main news article included either a negative or positive IAPS image $(615 \times 411$ pixels $)$, a matching heading, as well as a link to the entire news report. It is important to note that there was no other textual content regarding the main news. This was done to avoid attraction biases because of how appealing news may have been for individuals who participated. Since participants did not interact with the web pages, the link served no function, except for creating a realistic version of a news web page that can be found on the world wide web. The structure and content of the web pages remained the same throughout the whole study. However, the side of the negative and positive content was counterbalanced 

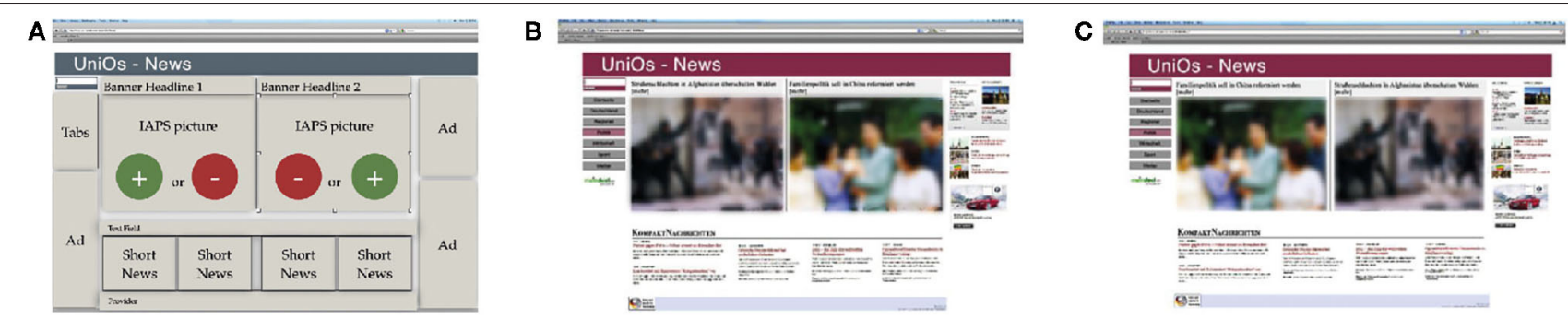

FIGURE 1 | Web pages used in the study. (A) Prototype web page with different sections: headlines, adds, tabs, news, and images. (B) Example of a web page with a negative image on the left side. (C) Example of a web page with a negative image on the right side [reverse of (B)].

(Figures 1B,C). The 48 images, embedded in the 24 web pages, differed from those used in the priming sessions.

The additional elements on each web page were four short news reports about ongoing current affairs. These elements were placed below the main news articles. The frame around the main news articles was completed by flanking advertisements on the left and right sides (Figure 1A). Please note that the statistical properties of forward directed saccades and backward directed saccades (regressions) while reading the text do not enter the analysis presented here in any form. As a standard feature on regular web pages, the upper left corner was secured for a tabs region which is necessary for general navigation. Previous work by Kaspar et al. (2015), using the same set of web pages, tested for the possibility that differences in eye movement parameters, within positive and negative images, could evolve from systematic differences in visual saliency. Therefore, a standard algorithm by Itti et al. (1998) that extracts the physical features of images and, based on this, predicts fixation patterns was applied. In addition to this, a graph-based visual saliency (GBVS) developed by Harel et al. (2007) was applied, as it predicts the fixations with a higher probability. After application, no difference regarding the visual saliency was found between the positive and negative images in the stimulus set [both $t_{(35)} \leq$ $0.941, p \geq 0.356]$.

\section{Procedure and Design}

We divided the participants randomly into two groups. One group started with the congruent block of the approachavoidance task. The other group started with the incongruent block. In each condition, participants faced a random sequence of 44 images of different valence ( 22 positive and 22 negative images). As soon as an image was presented, the participants had to respond with the joystick. The task paradigm required participants to push or pull the joystick in response to the image's valence. Participants used their dominant hand to manipulate the joystick in front of them. Participants in the congruent task condition had to pull (approach) the joystick toward themselves whenever a positive image was shown, and push (avoid) the joystick whenever a negative image was shown. In the incongruent condition, participants had to act reversely. They had to pull the negative images toward themselves and push away the positive ones (Figures 2B,C). They were instructed to respond as quickly and as accurately as possible.
It was not possible to rectify and correct response mistakes. Additionally, while moving the joystick toward or away, the image changed in size. The zoom feature of the approachavoidance task was programmed in MATLAB's Psychtoolbox V3 (r2017a; MathWorks Company); as such, a shown image smoothly decreased in size as soon as the joystick was pushed (Figure 2B). Conversely, the image size increased once the joystick was pulled (Figure 2C). It is important to note that participants were instructed to push or pull the joystick to its limit. Overall, participants took about $5 \mathrm{~min}$ to complete this first part of the experiment.

In the subsequent eye-tracking session, we recorded the viewing behavior on prototypes of 12 news web pages. Following earlier research of Kaspar et al. (2015) and ensuring the same experimental design, each web page was displayed for $15 \mathrm{~s}$. We instructed participants to explore the web pages freely (freeviewing task).

After the eye-tracking session, participants had a short break. The second part of the experiment, directly after the break, required the participants to complete the joystick approach-avoidance task in the other condition. Participants who performed in the congruent approach-avoidance task had to complete the incongruent condition. The opposite applied to participants in the other group. After the second priming session, an additional 12 web pages were displayed following the same procedure described above (free-viewing task).

\section{RESULTS}

\section{Performance in the Embodied Approach-Avoidance Task}

For the priming part of the experiment, we first calculated the accuracy of performance to check whether participants followed instructions. In the congruent condition, they had to pull positive and push negative primes. In the incongruent condition, the assigned actions were reversed. We found that participants made a low amount of errors (3.6\%). This suggests that instructions were clear, and participants followed them. Therefore, we excluded error trials from any further analysis.

Second, we focused on response times in the experiment's priming part to check whether positive and negative images in congruent and incongruent conditions involve different cognitive processes and, therefore, longer/shorter response times. 


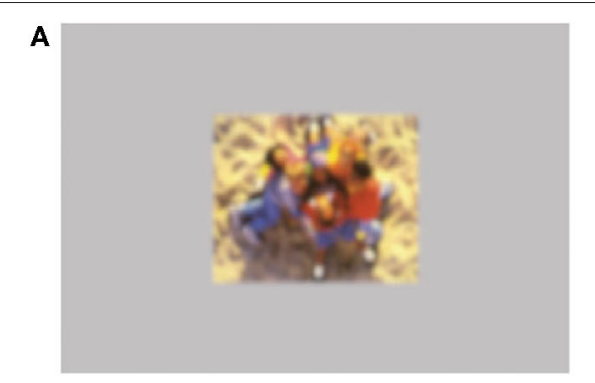

B

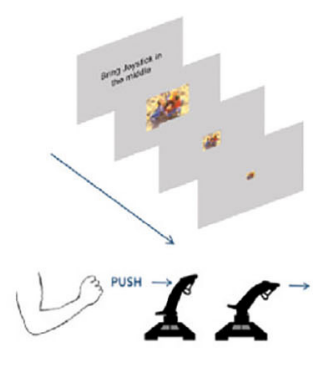

C

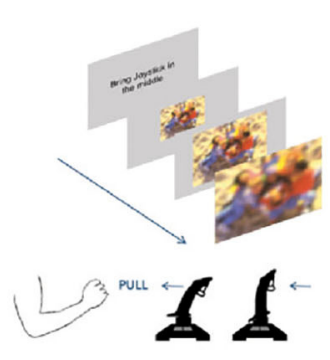

FIGURE 2 | Embodied primes used in the study. (A) Example of one priming stimulus: IAPS image with surrounding gray frame. This photograph depicts positive content with children laughing and playing on a sandy playground. Hint: Due to restrictions of showing IAPS images in public, it is masked with a diffusing filter. (B) Incongruent condition: joystick pushed forward-positive prime zooms out. (C) Congruent condition: joystick pulled backward-positive prime zooms in.

As the data showed a skewed distribution, we log-transformed it before further analysis (we used natural logarithm) (MarmolejoRamos et al., 2015). We used a linear mixed model (LMM) to analyze response times. The LMM was calculated with the lme4 package (Bates et al., 2014), and p-values were based on Wald's $T$-test using the lmerTest package (Kuznetsova et al., 2017). Degrees of freedoms were calculated using the Satterthwaite approximation. We modeled response times by image valence (positive and negative), and experimental condition (congruent and incongruent movements) as fixed effects and interactions between them. As random effects, we used random intercepts for grouping variable participants. For all predictors, we used effect coding scheme with binary factors coded as -0.5 and 0.5 . Thus, the resulting estimates can be directly interpreted as the main effects. This coding scheme's advantage is that the fixed effect intercept is estimated as the grand average across all conditions and not a baseline condition average. We found the main effect of the image valence $\left[t_{(1673.02)}=-3.726, p<0.001\right]$ on response times (Figure 3B). The natural logarithm of the response time to negative stimuli was about 0.049 times smaller than to the positive stimuli. This corresponds to a speedup (reduction of response time) by a factor of $5.03 \%$. Furthermore, we found the main effect of the congruency of the task $\left[t_{(1673.04)}=-4.71, p\right.$ $<0.001$; Figure 3A]. The response in the incongruent condition was slower by about $6.39 \%$. The interaction between these effects was not significant $\left[t_{(1673.02)}=-0.87, p>0.38\right]$. These results demonstrate independent additive effects of faster movements under congruent conditions and faster movements in response to negative pictures.

\section{Eye Movements in the Free-Viewing Task (Web Pages)}

As a next step, we investigated the effect of priming (condition: congruent and incongruent) on the viewing of news pages containing emotional stimuli (valence: negative and positive) on either side (side: left and right). The participants freely viewed different web pages containing one positive image and one negative image and additional filler texts, while we collected eye movement data. We characterized the exploration of these web pages with the two images as regions of interest (ROIs) with a various eye movement measures. Specifically, we used four different measures to quantify eye movements within ROIs: the average fixation duration within each image, the number of fixations within each image, the total dwell time on each image, and the length of saccades within each image. Additionally, we analyzed the number of saccades and their length from the outside to the inside of the images. For all six measures, we used the same statistical procedures. Similarly to the response time analysis, we employed linear mixed models. We modeled each of the variables by experimental condition (congruent and incongruent movements before the free-viewing task), image valence (positive and negative), and side of the image (left and right) as fixed effects and the interactions between them as random effects. We used random intercepts for grouping variable participants. For all predictors, we used effect coding scheme with binary factors coded as -0.5 and 0.5 . We visually inspected the normality of the data. All variables, aside from dwell time, were log-transformed to achieve normally distributed data. Jointly, these measures and analyses allow the characterization of viewing behavior on the web pages after priming.

\section{Fixation Duration Within ROIs}

As the first measure, we used the average fixation duration within each ROI to measure the depth of processing (Ehinger et al., 2018). We did not find the effect of condition $\left[t_{(8291.27)}=-0.615\right.$, $p=0.54]$ on fixation duration. However, we found the main effect of the valence $\left[t_{(8286.74)}=-3.513, p<0.001\right.$; Figure 4A] The average fixation duration on negative images was longer by about $2.8 \%$. Furthermore, we observed the main effect of the side $\left[t_{(8284.26)}=-3.093, p<0.01\right.$; Figure 4B]. Fixations on the image displayed on the left side were longer by about $2.45 \%$. Further, we found the significant interaction between valence and side $\left[t_{(8283.33)}=-3.318, p<0.001\right.$, Figure $\left.4 \mathrm{C}\right]$. The difference in fixation duration on positive and negative images was larger on the right side. The size of this interaction was of the same order of magnitude as the main effect of the side of the image. That is, the longest average fixation duration was observed for the combination of negative images on the right side of the displayed web page. All other two-way and threeway interactions were not significant $(p>0.18)$. These results show that the displayed web page parameters, i.e., valence and side, had a significant influence on the depth of processing at 


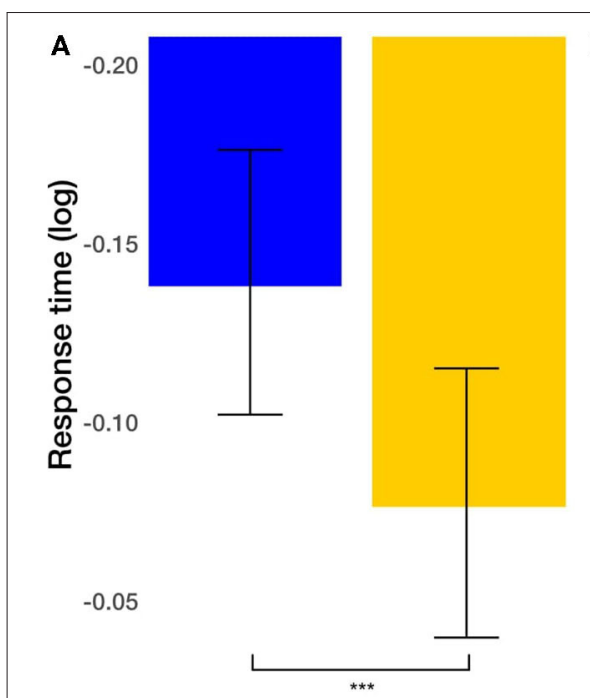

Congruent Incongruent

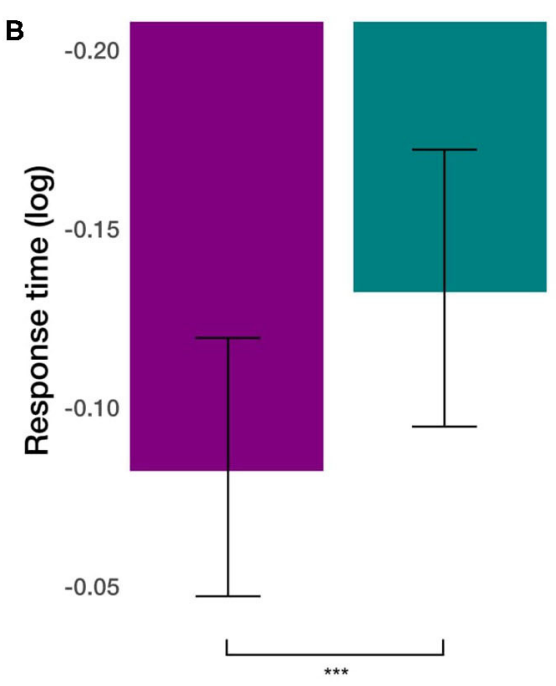

Negative
C

0.10

0.05

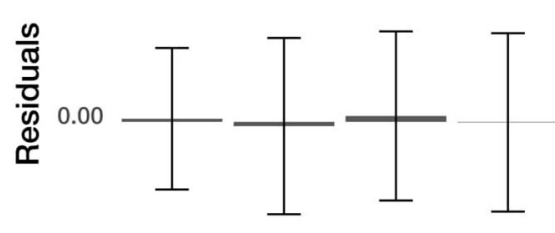

$-0.10$

FIGURE 3 | Response time results. (A) The main effect of the congruency of the priming. (B) The main effect of image valence (negative vs. positive). (C) Averaged residuals of all condition combinations from response time linear mixed model. In all plots, bars represent mean values and whiskers standard error of the mean. Stars represent significance levels: ${ }^{\star \star \star} p<0.001$.

individual fixation locations. However, this did not apply to the priming condition.

\section{Number of Fixations Within ROIs}

Next, we considered the number of fixations within each image to measure attention devoted to the respective stimulus. We found the main effect of condition $\left[t_{(915.2)}=-2.271, p=\right.$ 0.0234; Figure 5A]. The number of fixations within the ROIs after congruent priming was larger by $11.47 \%$. Furthermore, we observed the main effect of the valence $\left[t_{(915.19)}=-5.112, p\right.$ $<0.001$; Figure 5B]. Negative images captured 27.68\% more fixations than positive images. Finally, we found the main effect of the side $\left[t_{(915.17)}=-2.816, p<0.01\right.$; Figure $\left.5 \mathrm{C}\right]$. Images displayed on the left side captured $14.41 \%$ more fixations. All two-way and three-way interactions were not significant $(p>$ 0.1 ). These results demonstrate additive effects, in terms of the logarithm of the number of fixations. Converted back to the number of fixations within the ROIs, this results in multiplicative effects on the condition, valence, and side on the attention devoted to the images.

\section{Dwell Time Within ROIs}

The dwell time combines the aspects of fixation duration and the number of fixations within the ROIs. We found the main effect of condition $\left[t_{(915.2)}=-2.274, p=0.0232\right.$; Figure 6A]. The dwell time within the ROIs after congruent priming was on average $190 \mathrm{~ms}$ larger. Furthermore, we observed the main effect of the valence $\left[t_{(915.2)}=-6.146, p<0.001\right.$; Figure 6B]. Dwell time on negative images was on average $513 \mathrm{~ms}$ larger than on positive images. Finally, we observed the main effect of the side $\left[t_{(915.17)}=-3.381, p<0.01\right.$; Figure $\left.6 \mathrm{C}\right]$. On average, the dwell time within images was on average $282 \mathrm{~ms}$ larger on the left side. All two-way and three-way interactions were not significant $(p>0.33)$. These results resemble the results in the analysis of the number of fixations within ROIs. They provide evidence for independent effects of the priming condition, the valence of the viewed image, and the side of image location on the dwell time.

\section{Saccade's Length Within ROIs}

As a measure of exploration within the images, we used the saccadic length. We did not find the main effect of condition on the saccadic length $\left[t_{(8296.9)}=1.321, p=0.187\right]$. However, we did find the main effect of the image valence $\left[t_{(8291.31)}=4.253\right.$, $p<0.001$; Figure 7A]. Within negative images, saccades were shorter by $9.55 \%$. Further, we observed a small but significant main effect of the side $\left[t_{(8287.02)}=2.618, p<0.01\right.$; Figure $\left.7 \mathbf{B}\right]$ on the saccade's length. Saccades were shorter by $5.76 \%$ on the left side. Furthermore, we found significant two-way interactions between the image valence and the side of the image $\left[t_{(8285.35)}=\right.$ $-2.038, p=0.0415$; Figure 7C], with a slightly larger difference in the saccadic length for positive and negative images on the left side. Additionally, we observed the interaction of condition and side $\left[t_{(8289.3)}=2.907, p<0.01\right.$; Figure 7D]. Whereas, images displayed on the left condition were trivial, images on the right side were explored by longer saccades after incongruent priming. We did not find the interaction between condition and valance $(p>0.96)$, as well as no three-way interaction between all factors $(p>0.27)$. These results give evidence for a more focused 
A

540

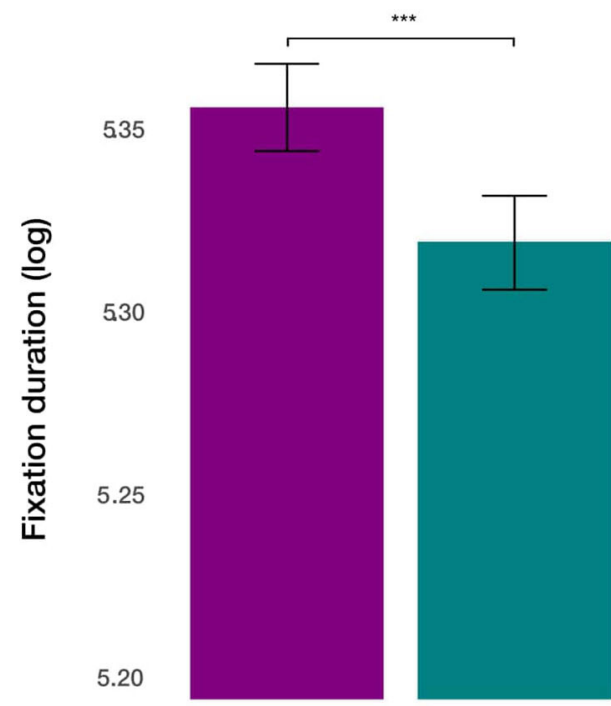

C

Negative

540

535

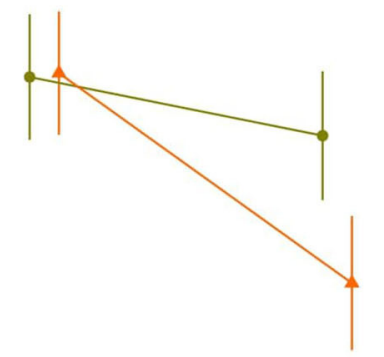

5.25
B

540

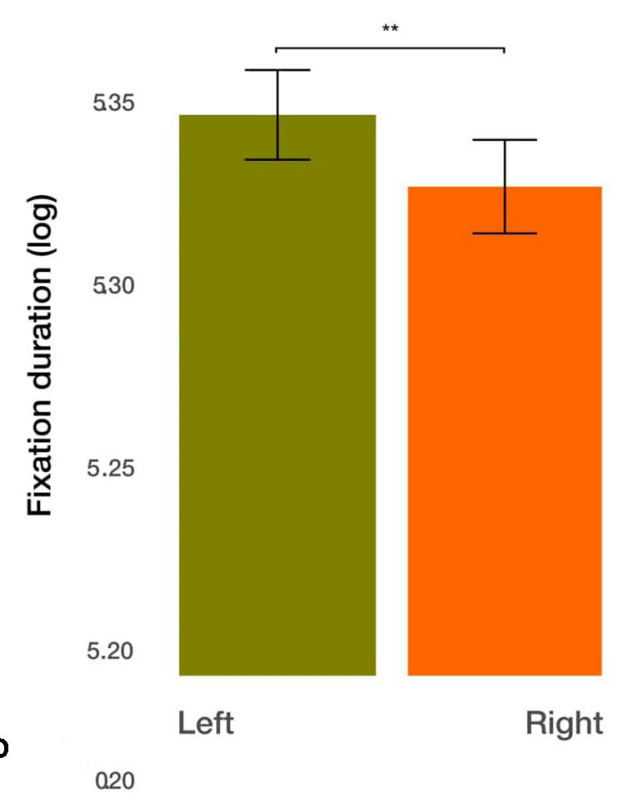

0.10

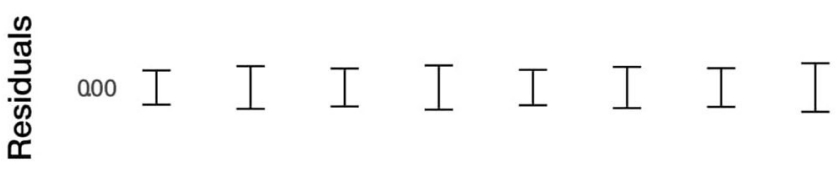

$-010$

Left

Right

Congruent Incongruent Congruent Incongruent

Negative Positive Negative Positive Negative Positive Negative Positive

\section{Negative Positive}

FIGURE 4 | Fixation duration results. (A) The main effect of image valence (negative vs. positive). (B) The main effect of the side (left vs. right). (C) The interaction between valence and side. (D) Averaged residuals of all condition combinations from the fixation duration linear mixed model. In all plots, bars represent mean values and whiskers standard error of the mean. Stars represent significance levels: ${ }^{\star \star} p<0.01$ and ${ }^{* \star *} p<0.001$.

exploration of images with negative valence, specifically when displayed on the left side. The priming condition modulated the influence of the side with a larger differential effect on the exploration of images displayed on the right side.

\section{Number and Length of Saccades From Outside ROls Toward ROls}

As a measure of how well the images can attract attention, we utilized the number of saccades from outside the image toward the inside. We found a trend toward significance for the main effect of the congruency of the priming $\left[t_{(850.76)}=\right.$ $-1.894, p=0.0586]$. Participants, on average, made $6.96 \%$ more saccades from outside into an image after congruent condition. The main effect of the valence of the image $\left[t_{(851.31)}\right.$ $=-1.936, p=0.0532]$ on the number of saccades from outside images toward them missed the significance threshold. Nominally, participants made $7.13 \%$ more saccades on negative images. The effect of the side of the image and all two-way 


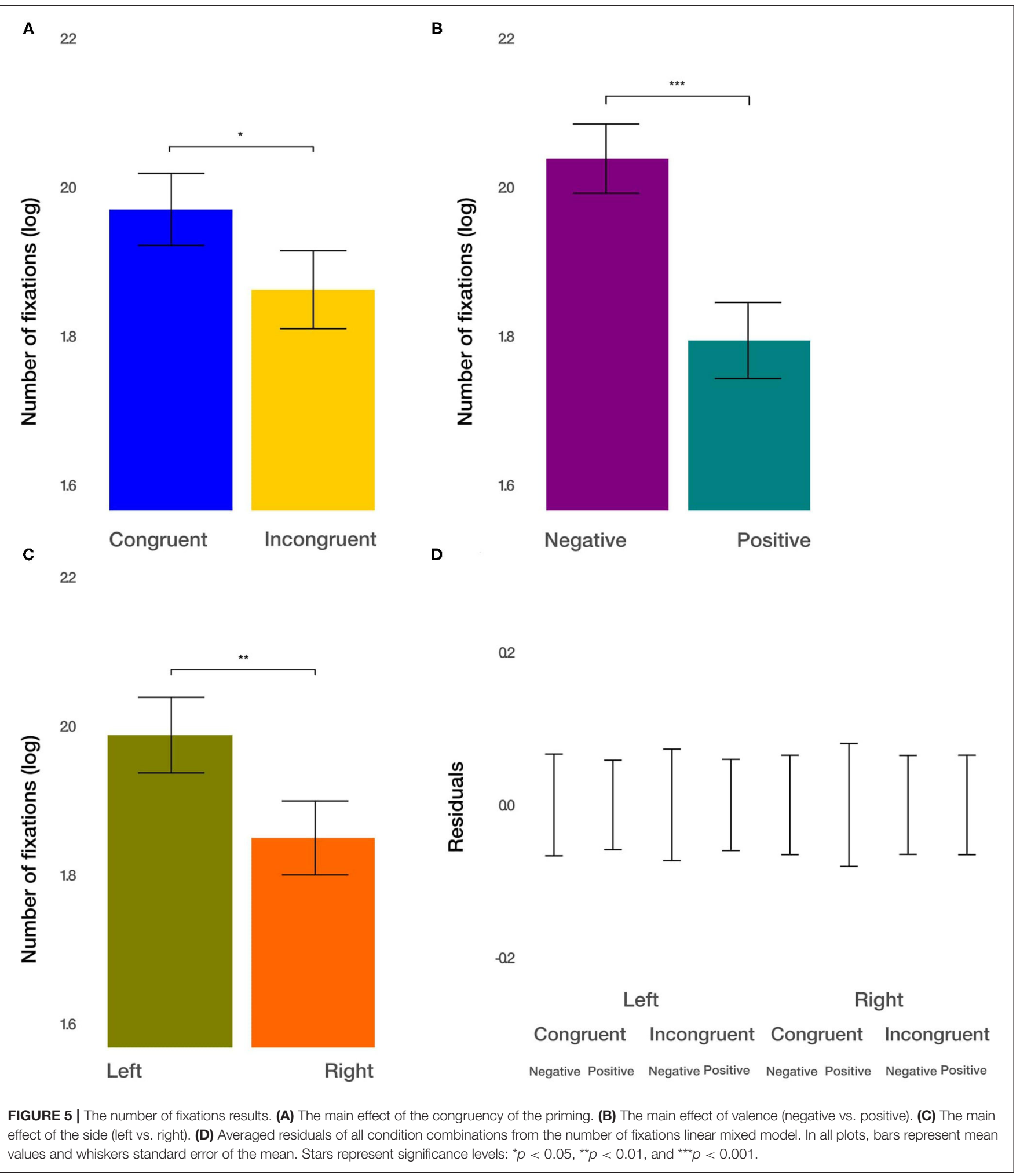

and three-way interactions were not significant (all $p>0.12$ ). Furthermore, we analyzed the length of saccades from outside images toward them. We found the main effect of congruency of the priming $\left[t_{(2011.69)}=2.189, p=0.0287\right]$. After priming with congruent actions, the average saccade length from outside into the images was shorter by $9.71 \%$. Furthermore, the main effect of the side of the image $\left[t_{(2009.88)}=2.269, p=0.0234\right)$ on the saccade's length from outside images toward them was 


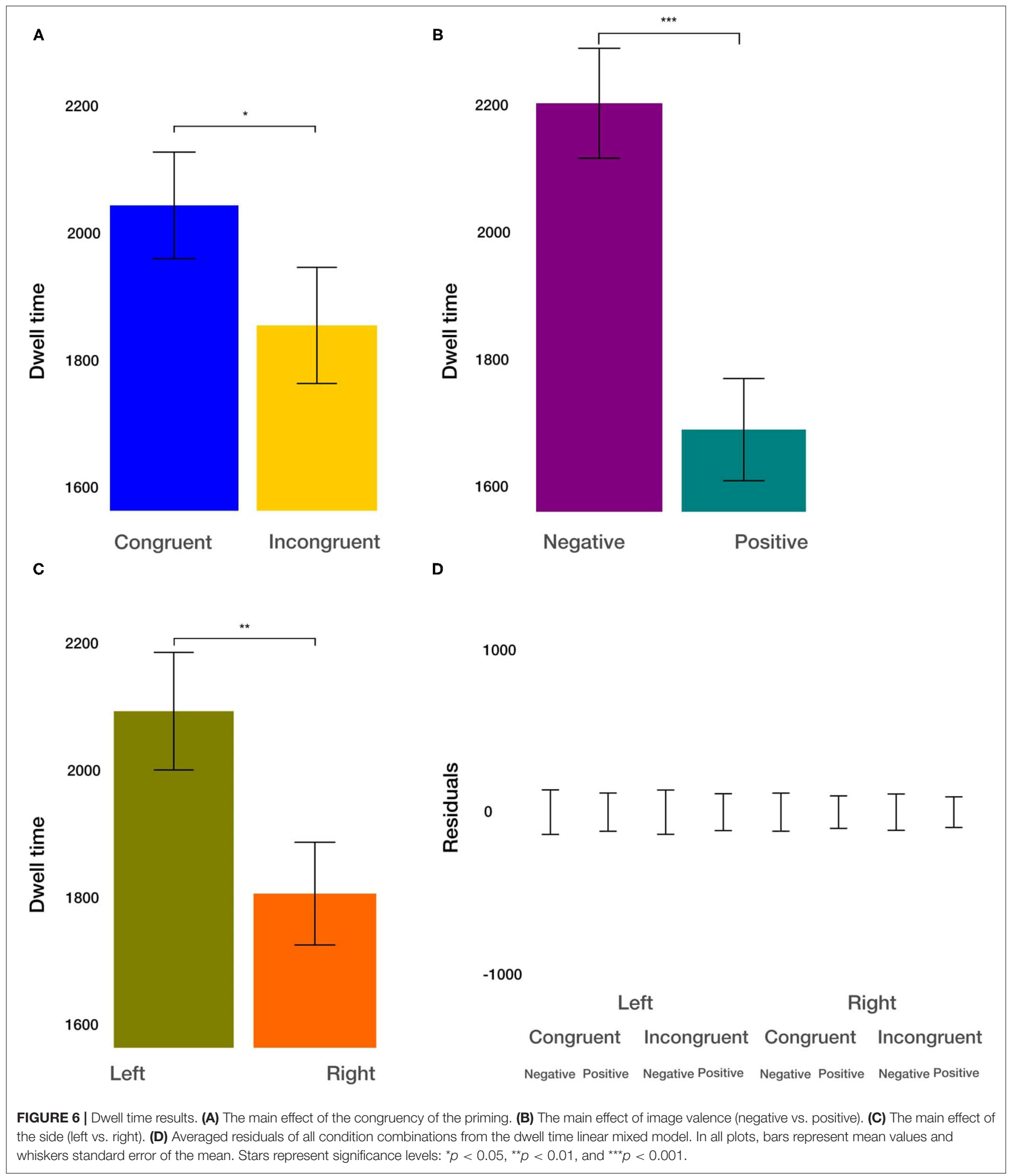

significant. Saccades targeting the left image were, on average, shorter by $10.07 \%$. The effect of the image valence and all two-way and three-way interactions were not significant (all $p>$ $0.06)$. These results suggest that participants, on average, make shorter and more saccades after congruent priming from outside images toward them. Furthermore, on average, participants make longer saccades from outside images toward the images on the right side. 


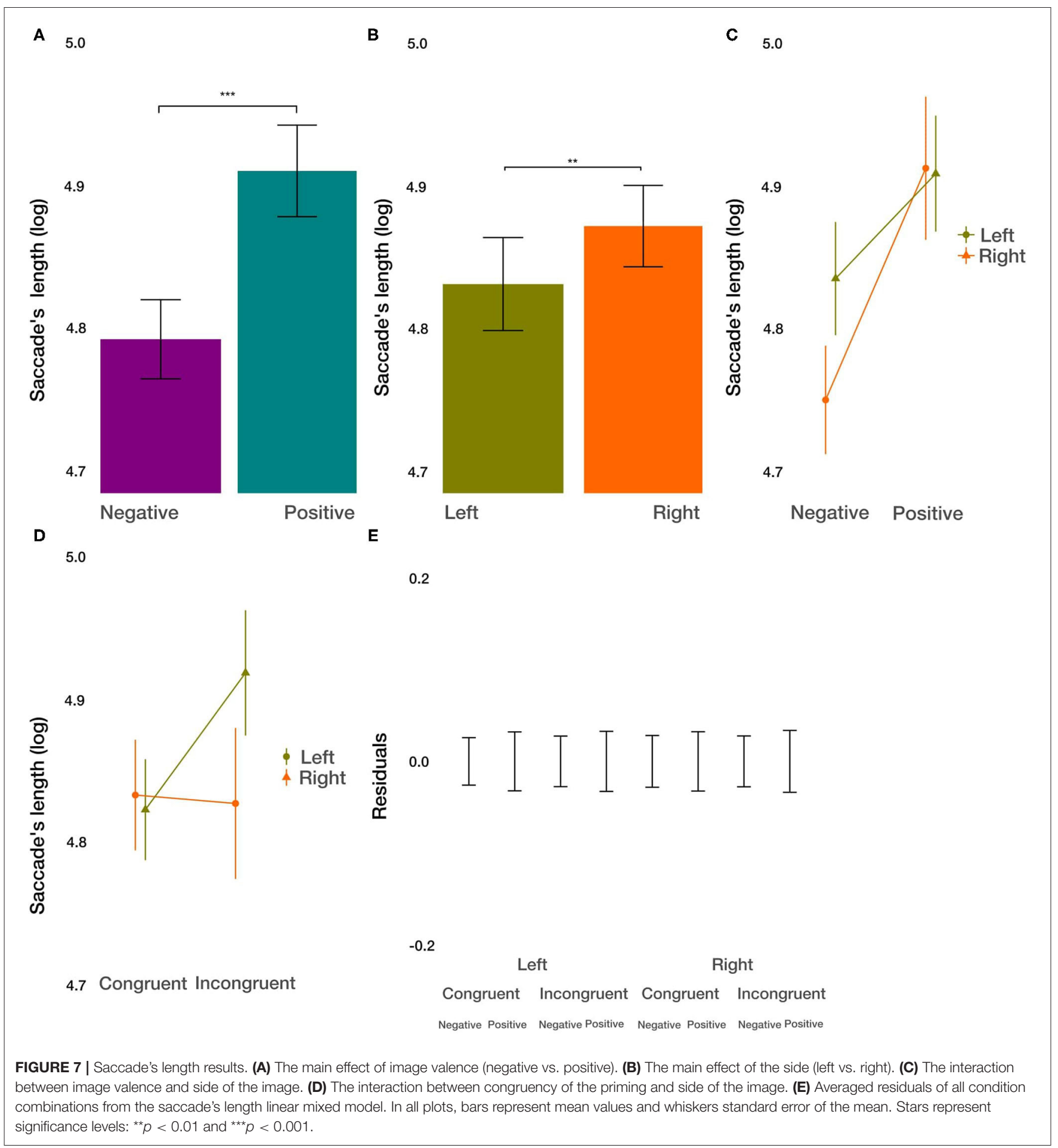

\section{DISCUSSION}

In the present study, we used an active bodily interaction with affective stimuli in an approach-avoidance task to investigate the influence on the later free visual exploration of news web pages containing emotional images. First, positively or negatively valenced images were zoomed in or out by pulling or pushing the joystick. Here we found multiplicative effects of valence and condition. That is, we could replicate the results of previous studies and report a faster response in the congruent condition. Furthermore, negative stimuli were reacted to faster. The lack of interaction and the additive effects on the log-response time suggest independent multiplicative effects on the base response time. Second, concerning the influence of embodied 


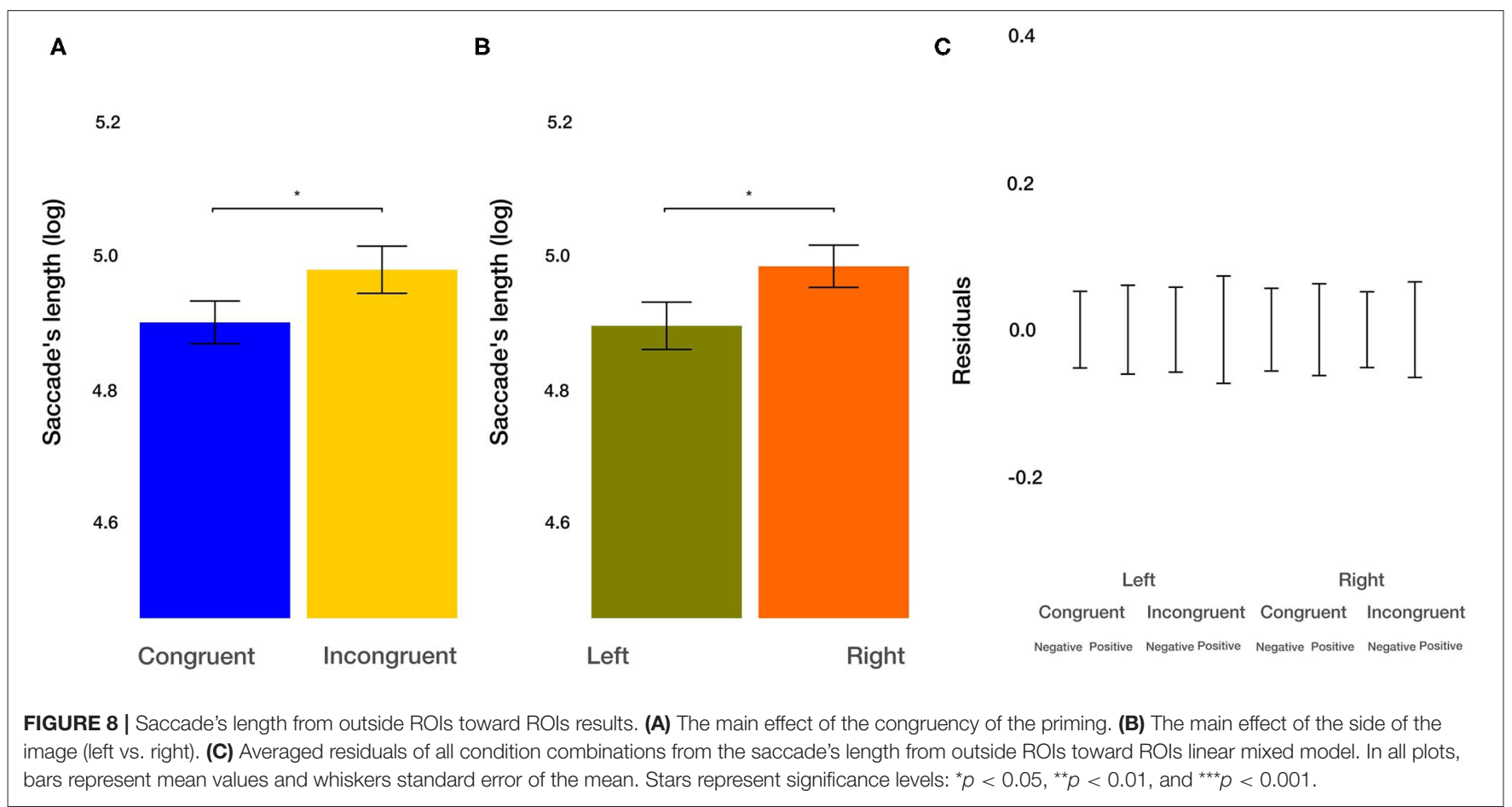

priming on eye movements in the subsequent free-viewing task, we observed the main effects of valence, side of the presentation, condition, as well as specific interactions. This demonstrates the influence of stimulus properties (valence), internal variables (priming by condition), and spatial properties (side) on visual exploration.

The following discussion will address the two main parts of our analyses. We will first discuss the viewing behavior (fixations and saccades) made only in the emotion-laden main news. The subsequent part deals with the approach and avoidance behavior while performing the approach-avoidance task while taking the response times into account.

First, we observed an influence of the priming condition, i.e., performing congruent or incongruent actions in the approachavoidance task on later visual exploration. Specifically, after priming in the congruent condition, the number of fixations on images increased, the total dwell time increased, and the average saccade length from outside of the images toward the images decreased. The combination of these effects suggests increased attention to web pages' image content after the subjects performed congruent actions on images in the priming phase. Thus, congruent bodily interaction with images in the priming phase fosters visual interaction in the subsequent exploration phase.

Second, we found systematic effects of the valence of images in the exploration phase. Specifically, on images with negative valence, the average fixation duration was prolonged, more fixations were completed, and the total dwell time increased. Particularly, the average length of saccades within the images of negative valence decreased. This combination of effects speaks for increased scrutiny of negative valenced images.
Third, we observed a lateral asymmetry in the visual exploration phase. On average, participants displayed longer fixations, more fixations, and longer dwell time on the left side. The change of the length of saccades within the images was significant but quantitatively not relevant. In contrast, the saccades' length from the outside of the images toward the inside was shorter on the left side. The combination of these effects largely resembles the effects observed with respect to the priming condition and suggests increased visual interaction with stimuli presented on the left side. These results further corroborate results suggesting spatial biases in eye movements (Ossandon et al., 2014). This suggests that biases toward the left side influence not only the number of fixations but the major properties of visual exploration as well.

Finally, with respect to interactions of condition, valence, and side, it is noteworthy that there were only a few. For the number of fixations, dwell time, and the average length of saccades from outside to inside, we did not observe any 2-way or 3-way interactions, and the residuals after discounting for the main effects were relatively small (Figures 3C, 4D, 5D, 6D, 7E, 8). Only for the average saccadic length within the images did we observe an interaction of valence ${ }^{\star}$ side and condition*side and for the average fixation duration an interaction of valence* side. It appears that with respect to the saccadic length within the images for the left side, the valence is more important, and the priming condition less important for images on the left side. The fixation duration is less affected by image valence on the left side. Overall, it is striking that the effects of the three independent variables are largely independent, and the interactions are limited to a few aspects.

The results of this study suggest that approach and avoidance reactions in humans have a direct influence on attention 
allocation and gaze behavior. We used the embodied cognition approach and, more specifically, the approach-avoidance task to explore its effect on eye movements. This study adds to the limited amount of eye-tracking research that has dealt with the interplay of top-down influences and bottom-up features.

To induce a positive or a negative emotional state, Kaspar et al. (2015) had participants watch either positive or negative sequences of 44 full-colored images from the International Affective Picture System (IAPS; Lang et al., 1997) with a valence rating below 3 for negative and a valence rating above 7 for positive primes. In the subsequent eye-tracking session, they presented 24 similarly structured webpages that included a positive and a negative IAPS image: one on the left, the other on the right. They found that a negative emotional state marginally elicited a more spatially extensive exploration. In our study, we used the same news web pages. However, instead of inducing emotional states by passively watching pictures, we used an approach-avoidance task as an embodied prime for positive and negative emotional states. In contrast, no specific emotional valence was primed, but rather the congruent or incongruent action, i.e., approach/avoidance of positive/negative valenced stimuli or the reverse assignment. There is ample evidence that our emotions affect our visual behavior. Regarding the direct effect of emotions on visual exploration, the broaden-and-build model of positive emotions (Fredrickson, 1998) claims that positive emotions such as joy, interest, elation, or love, temporarily expand the focus of attention, therefore, increasing the thought-action repertoire by fostering interest in the environment and encouraging play and exploration (Fredrickson, 2000). Accordingly, being in a happy emotional state vs. being in a sad or neutral emotional state has been shown to increase participants' breadth of attention (Rowe et al., 2007). Similarly, Wadlinger and Isaacowitz (2006) found that the distribution of participants' fixations on an image is broader in individuals induced into a positive emotional state, with more frequent saccades to neutral or positively valenced parts and with more fixations on positively valenced peripheral stimuli. Whereas, broadened attention is often associated with anxiety (Gruzelier and Phelan, 1991), which has led some to speculate that this might be an adaption to a negative emotional state (Garland et al., 2011), while a positive emotional state may reduce the motivation to scrutinize the environment because of an increased feeling of safety (Schwarz, 1990). Part of the explanation for these diverse findings may be that the emotional state induction procedures are also diverse, particularly concerning neutral emotional states. For instance, whereas some actively induce a positive emotional state by offering participants a bag of candies but simply do nothing in the neutral condition (Wadlinger and Isaacowitz, 2006), others rely on a waiting room manipulation to actively induce a neutral emotional state (Herz et al., 2004). Others have also been known to use movies (Grubert et al., 2013) or music (Shapiro and Lim, 1989). According to Kaspar et al. (2015), this diversity of emotional state induction method questions the assumption that a neutral emotional state is always an adequate control condition. This may help explain why being in a negative emotional state had the same effect as being in a neutral emotional state according to some studies (Rowe et al., 2007). Whereas, other studies found similar effects of being in a positive and neutral emotional state (Chipchase and Chapman, 2013). In light of this still unresolved issue, the present study followed (Kaspar et al., 2015) and solely contrasted positive with negative emotional states and focused on the effects of priming in congruent vs. incongruent actions in an approach-avoidance task.

When investigating embodied cognition, a high degree of ecological validity is necessary. We instructed participants to use a joystick to either approach or avoid positively or negatively valenced pictures displayed on a screen (Ernst et al., 2013). To increase immersion, we implemented a visual "zooming-effect" (Rinck and Becker, 2007). When the joystick was pushed, the images were zoomed out, and when it was pulled, the images were zoomed in. This not only ensured a more realistic impression of movement toward or away from the images, but it also illustrated any ambiguity in the participants' arm movements. The appraisal of a movement depends upon what is achieved (Lavender and Hommel, 2007; Krieglmeyer et al., 2010). Stretching out one's arm often indicates a negatively valenced avoidance-behavior, i.e., when a harmful object is pushed away. Yet, it can also be an indispensable part of a positively valenced approach-behavior, for instance when one reaches for nourishing food or one's infant. A joystick-based Approach avoidance task with a zooming-effect resolves this ambivalence. To further increase the immersion utilizing techniques of virtual reality offer themselves.

In addition to the effect of participants' emotional states on their attention, we also explored the approach and avoidance behavior in the priming conditions. Since the IAPS pictures have exhibited an impact on the emotions of participants and therefore serve as a reliable priming method (Kaspar et al., 2015), we made use of them in our study to also modulate congruent vs. incongruent actions by the participants. Previous study designs have solely presented participants with a row of pictures within one category. However, our study design differs from previous work in that we let the participants visually and physically interact with the depicted pictures. For this reason, we joined pictures of two valence categories in one task, which had to be treated differently. Since we were working with IAPS images, it is worthy of mentioning that the highly negative images were also accompanied by a higher level of arousal, in contrast to highly positive images (Lang et al., 1997). This applies to the IAPS images within the priming block and the images embedded in the news web pages. As Kaspar et al. (2015) note, negative emotions, such as anxiety, anger, and fear, also happen to be more arousing for the participants compared to positive emotions, such as pride or happiness. This applies as well to negative and positive emotional conditions. As mentioned in the introduction, along with the increment of arousal in negative emotions comes an increase in attention. This is caused by the initiation of survivalrelated actions related to behavioral and physical fight-or-flight responses (Fredrickson, 2000). In turn, the specific arousal, which is immediately elicited by the mere presence of the valenced images, is interwoven with the arousal that is elicited by the interactive treatment of the images in the priming condition. Since the primes used in this study comprised of both valence categories, it is challenging to make any explicit distinction. 
However, the approach-avoidance task served as an authentic method to strive for and impact the internal approach and avoidance reactions in humans. Participants in the incongruent priming condition were significantly slower in treating the primes as instructed. Thus, they were slower to pull negative images toward themselves and push positive images away from themselves. The difficulty of the incongruent priming condition task was reflected in the participants' response times. In general, the task instruction (to pull negative images toward oneself) essentially acts against the avoidance effect, which has been presented as an example of embodied cognition that emphasizes action-oriented behavior, i.e., actions related to survival. A direct comparison of both task conditions clearly revealed the avoidance effect. In the congruent condition, participants were significantly faster to avoid the negative stimuli, compared with avoiding the positive stimuli in the incongruent condition.

In controlled attentional shifts, older adults show a positivity bias and negativity avoidance (Isaacowitz et al., 2006). In contrast, no such bias is observable for automatic attentional shifts (Hahn et al., 2006; Mather and Knight, 2006; Knight et al., 2007). The results are inconclusive for younger adults. Some studies find a preference for negative stimuli (Thomas and Hasher, 2006; Tomaszczyk et al., 2008), while others report a tendency to avoid negative stimuli (Becker and Detweiler-Bedell, 2009). Some studies find emotional stateincongruent preferences (Parrott and Sabini, 1990; Schwager and Rothermund, 2013), while others report emotional statecongruent preferences (Ferraro et al., 2003; Isaacowitz et al., 2008; Koster et al., 2010; Becker and Leinenger, 2011). Presumably, this inconsistency is partly because studies only focused on external affective influences and disregarded the participants' emotional state. However, considering the participants' emotional states is crucial because one's emotional state can determine one's current goals. In fact, when emotional regulation is the primary goal of younger adults, they focus less on negative images and more on positive images (Xing and Isaacowitz, 2006). Moreover, students who learn to focus on positive stimuli subsequently show reduced attention for negative stimuli (Wadlinger and Isaacowitz, 2006), indicating that attention is a powerful tool for emotional states regulation (Wadlinger and Isaacowitz, 2011). In contrast, Das and Fennis (2008) found that a positive emotional state can increase attention for negative information. However, the primary goal of young adults exploring news pages is arguably not emotional state regulation. They are rather in a "browsing mode" in which they search for personally interesting information. In such a mode, features of the stimulus, such as its valence, are more likely to catch the observer's attention (Hamborg et al., 2012). In contrast to these mixed results, the effects of congruent vs. incongruent conditions in the present study are relatively straightforward.

Many studies investigated an automatic approach bias in patients with substance abuse disorders. Individuals with a substance abuse disorder exhibit an automatic bias toward drugrelated words (Cox et al., 2006) or pictures (Field et al., 2013). In stimulus-response compatibility tasks, in which participants have to use a joystick to move cues either away or toward themselves, they approach rather than avoid drug-related cues and they approach them faster than they avoid them. In an implicit approach-avoidance task, in which participants push and pull cues according to formal features [like the format of a picture (Wiers et al., 2011) or its vertical alignment (Cousijn et al., 2011)], heavy drinkers (Wiers et al., 2009), patients with alcohol abuse disorder (Wiers et al., 2011, 2014), heroin addicts (Zhou et al., 2012), smokers (Wiers C.E. et al., 2013; Wiers R.W. et al., 2013a,b), and cannabis users (Cousijn et al., 2011) approach drug cues faster than healthy controls. In an explicit approachavoidance task in which participants either push away drug cues while pulling neutral cues toward them or vice versa, individuals with alcohol abuse disorder approach drug cues faster than they avoid them (Ernst et al., 2014). Thus, there is accumulating evidence for a general automatic approach/avoidance bias related to substance abuse.

Essentially, with our empirical work we cannot address the dispute on causal and constitutive relationships (Kaiser and Krickel, 2017). In the spirit of hypothesis testing we present data, that are compatible with the framework of embodied cognition. Our results could be further explained by two different aspects (limitations) that we address here. First, one of the limitations is that we do not know whether embodied priming with emotionally laden pictures triggered any emotions. One could test that by measuring different levels of arousal during the priming phase. However, we did not investigate the exact physiological basis behind embodied priming but its influence on the viewing behavior. In the future, it would be worth also studying these physiological underpinnings of embodied priming. Second, we used binary categories (positive and negative images) in our study based on the validated dataset (Lang et al., 1997). Emotions and perception of emotionally laden images can vary between participants, and therefore, one could additionally improve understanding how the effect that we found emerged if participants rated the imaged themselves. These issues do not change our interpretation, but it is essential to consider them.

In summary, we present how congruent embodied priming influences eye movements in a free-viewing task. Results presented in our study suggest that prior congruent movements, in line with our bodily reactions, can influence how we scrutinize images presented on the World Wide Web.

We found that movements in line with our bodily reactions (approach positive and avoid negative) influence how we observe images presented on the World Wide Web.

\section{DATA AVAILABILITY STATEMENT}

The datasets presented in this study can be found in online repositories. The names of the repository/repositories and accession number(s) can be found in the article/supplementary material.

\section{ETHICS STATEMENT}

The studies involving human participants were reviewed and approved by Ethics committee of the Osnabrück University. The 
patients/participants provided their written informed consent to participate in this study.

\section{AUTHOR CONTRIBUTIONS}

PK and SW: conceived the study. FA and PK: study design. FA: data collection. AC, FA, and PK: data analysis and revisions and finalizing the manuscript. AC: initial draft of the manuscript. All authors contributed to the article and approved the submitted version.

\section{REFERENCES}

Barsalou, L. W. (2008). Grounded cognition. Annu. Rev. Psychol. 59, 617-645. doi: 10.1146/annurev.psych.59.103006.093639

Bates, D., Mächler, M., Bolker, B., and Walker, S. (2014). Fitting linear mixedeffects models using lme4. arXiv:1406.5823 [stat]. doi: 10.18637/jss.v067.i01

Becker, M. W., and Detweiler-Bedell, B. (2009). Short article: early detection and avoidance of threatening faces during passive viewing. Q. J. Exp. Psychol. 62, 1257-1264. doi: 10.1080/17470210902725753

Becker, M. W., and Leinenger, M. (2011). Attentional selection is biased toward mood-congruent stimuli. Emotion 11, 1248-1254. doi: 10.1037/a0023524

Bhalla, M., and Proffitt, D. R. (1999). Visual-motor recalibration in geographical slant perception. J. Exp. Psychol. 25:1076. doi: 10.1037/0096-1523.25.4.1076

Brown, E. C., Tas, C., Kuzu, D., Esen-Danaci, A., Roelofs, K., and Brüne, M. (2014). Social approach and avoidance behaviour for negative emotions is modulated by endogenous oxytocin and paranoia in schizophrenia. Psychiatry Res. 219, 436-442. doi: 10.1016/j.psychres.2014.06.038

Casasanto, D., and Dijkstra, K. (2010). Motor action and emotional memory. Cognition 115, 179-185. doi: 10.1016/j.cognition.2009.11.002

Chen, M., and Bargh, J. A. (1999). Consequences of automatic evaluation: immediate behavioral predispositions to approach or avoid the stimulus. Pers. Soc. Psychol. Bull. 25, 215-224. doi: 10.1177/0146167299025002007

Chipchase, S. Y., and Chapman, P. (2013). Trade-offs in visual attention and the enhancement of memory specificity for positive and negative emotional stimuli. Q. J. Exp. Psychol. 66, 277-298. doi: 10.1080/17470218.2012.707664

Colombetti, G. (2014). The Feeling Body: Affective Science Meets the Enactive Mind. Cambridge, MA: MIT Press. doi: 10.7551/mitpress/9780262019958.001.0001

Cousijn, J., Goudriaan, A. E., and Wiers, R. W. (2011). Reaching out towards cannabis: approach-bias in heavy cannabis users predicts changes in cannabis use: approach-bias and cannabis use. Addiction 106, 1667-1674. doi: 10.1111/j.1360-0443.2011.03475.x

Cox, W. M., Fadardi, J. S., and Pothos, E. M. (2006). The addiction-stroop test: theoretical considerations and procedural recommendations. Psychol. Bull. 132, 443-476. doi: 10.1037/0033-2909.132.3.443

Damasio, A. (2001). Fundamental feelings. Nature 413, 781-781. doi: $10.1038 / 35101669$

Damasio, A. R. (1999). The Feeling of What Happens: Body and Emotion in the Making of Consciousness. Houghton Mifflin Harcourt.

Das, E., and Fennis, B. M. (2008). In the mood to face the facts: when a positive mood promotes systematic processing of self-threatening information. Motiv. Emot. 32, 221-230. doi: 10.1007/s11031-008-9093-1

Ehinger, B. V., Kaufhold, L., and König, P. (2018). Probing the temporal dynamics of the exploration-exploitation dilemma of eye movements. J. Vis. 18:6. doi: 10.1167/18.3.6

Einhäuser, W., Spain, M., and Perona, P. (2008). Objects predict fixations better than early saliency. J. Vis. 8:18. doi: $10.1167 / 8.14 .18$

Einhäuser, Wolfgang, U. R. and Koch, C. (2008). Task-demands can immediately reverse the effects of sensory-driven saliency in complex visual stimuli. J. Vis. 8:2. doi: $10.1167 / 8.2 .2$

Engel, A. K., Maye, A., Kurthen, M., and König, P. (2013). Where's the action? The pragmatic turn in cognitive science. Trends Cogn. Sci. 17, 202-209. doi: $10.1016 /$ j.tics.2013.03.006

Ernst, L. H., Plichta, M. M., Dresler, T., Zesewitz, A. K., Tupak, S. V., Haeussinger, F. B., et al. (2014). Prefrontal correlates of approach preferences for alcohol

\section{FUNDING}

We gratefully acknowledge the support by the DFG-funded Research Training Group Situated Cognition (GRK 2185/1), Niedersächsischen Innovationsförderprogramms für Forschung und Entwicklung in Unternehmen (NBank)-EyeTrax, and the Deutsche Forschungsgemeinschaft (DFG) Open Access Publishing Fund of Osnabrück University. Moreover, we would like to thank Clayton Thompson for feedback on the manuscript.

stimuli in alcohol dependence: approach bias for alcohol. Addict. Biol. 19, 497-508. doi: 10.1111/adb. 12005

Ernst, L. H., Plichta, M. M., Lutz, E., Zesewitz, A. K., Tupak, S. V., Dresler, T., et al. (2013). Prefrontal activation patterns of automatic and regulated approachavoidance reactions-A functional near-infrared spectroscopy (fNIRS) study. Cortex 49, 131-142. doi: 10.1016/j.cortex.2011.09.013

Ferraro, F. R., King, B., Ronning, B., Pekarski, K., and Risan, J. (2003). Effects of induced emotional state on lexical processing in younger and older adults. $J$. Psychol. 137, 262-272. doi: 10.1080/00223980309600613

Field, M., Mogg, K., Mann, B., Bennett, G. A., and Bradley, B. P. (2013). Attentional biases in abstinent alcoholics and their association with craving. Psychol. Addict. Behav. 27, 71-80. doi: 10.1037/a0029626

Fredrickson, B. L. (1998). What good are positive emotions? Rev. Gen. Psychol. 2, 300-319. doi: 10.1037/1089-2680.2.3.300

Fredrickson, B. L. (2000). Cultivating positive emotions to optimize health and well-being. Prevent. Treat. 3:1a. doi: 10.1037/1522-3736.3.1.31a

Fridland, E., and Wiers, C. E. (2018). Addiction and embodiment. Phenomenol. Cogn. Sci. 17, 15-42. doi: 10.1007/s11097-017-9508-0

Garland, E. L., Gaylord, S. A., and Fredrickson, B. L. (2011). Positive reappraisal mediates the stress-reductive effects of mindfulness: an upward spiral process. Mindfulness 2, 59-67. doi: 10.1007/s12671-011-0043-8

Glenberg, A. M., and Kaschak, M. P. (2002). Grounding language in action. Psychon. Bull. Rev. 9, 558-565. doi: 10.3758/BF03196313

Grubert, A., Schmid, P., and Krummenacher, J. (2013). Happy with a difference, unhappy with an identity: observers' mood determines processing depth in visual search. Attent. Percept. Psychophys. 75, 41-52. doi: 10.3758/s13414-012-0385-x

Gruzelier, J., and Phelan, M. (1991). Stress induced reversal of a lexical divided visual-field asymmetry accompanied by retarded electrodermal habituation. Int. J. Psychophysiol. 11, 269-276. doi: 10.1016/0167-8760(91)90021-O

Hahn, S., Carlson, C., Singer, S., and Gronlund, S. D. (2006). Aging and visual search: automatic and controlled attentional bias to threat faces. Acta Psychol. 123, 312-336. doi: 10.1016/j.actpsy.2006.01.008

Hamborg, K.-C., Bruns, M., Ollermann, F., and Kaspar, K. (2012). The effect of banner animation on fixation behavior and recall performance in search tasks. Comput. Hum. Behav. 28, 576-582. doi: 10.1016/j.chb.2011. 11.003

Harel, J., Koch, C., and Perona, P. (2007). "Graph-based visual saliency," in Advances in Neural Information Processing Systems 19 (NIPS 2006) (Cambridge, MA: MIT Press), 545-552.

Hayhoe, M. M., Shrivastava, A., Mruczek, R., and Pelz, J. B. (2003). Visual memory and motor planning in a natural task. J. Vis. 3:6. doi: 10.1167/3.1.6

Herz, R. S., Schankler, C., and Beland, S. (2004). Olfaction, emotion and associative learning: effects on motivated behavior. Motiv. Emot. 28, 363-383. doi: 10.1007/s11031-004-2389-x

Heuer, K., Rinck, M., and Becker, E. S. (2007). Avoidance of emotional facial expressions in social anxiety: the approach-avoidance task. Behav. Res. Ther. 45, 2990-3001. doi: 10.1016/j.brat.2007.08.010

Hommel, B., Chapman, C. S., Cisek, P., Neyedli, H. F., Song, J.-H., and Welsh, T. N. (2019). No one knows what attention is. Attent. Percept. Psychophys. 81, 2288-2303. doi: 10.3758/s13414-019-01846-w

Isaacowitz, D. M., Toner, K., Goren, D., and Wilson, H. R. (2008). Looking while unhappy: mood-congruent gaze in young adults, positive gaze in older adults. Psychol. Sci. 19, 848-853. doi: 10.1111/j.1467-9280.2008.02167.x 
Isaacowitz, D. M., Wadlinger, H. A., Goren, D., and Wilson, H. R. (2006). Is there an age-related positivity effect in visual attention? A comparison of two methodologies. Emotion 6, 511-516. doi: 10.1037/1528-3542.6.3.511

Itti, L., and Koch, C. (2000). A saliency-based search mechanism for overt and covert shifts of visual attention. Vis. Res. 40, 1489-1506. doi: 10.1016/S0042-6989(99)00163-7

Itti, L., Koch, C., and Niebur, E. (1998). A model of saliency-based visual attention for rapid scene analysis. IEEE Trans. Pattern Anal. Mach. Intell. 20, 1254-1259. doi: $10.1109 / 34.730558$

Kaiser, M. I., and Krickel, B. (2017). The metaphysics of constitutive mechanistic phenomena. Br. J. Philos. Sci. 68, 745-779. doi: 10.1093/bjps/axv058

Kaspar, K., Gameiro, R. R., and König, P. (2015). Feeling good, searching the bad: positive priming increases attention and memory for negative stimuli on webpages. Comput. Hum. Behav. 53, 332-343. doi: 10.1016/j.chb.2015. 07.020

Kaspar, K., Hloucal, T.-M., Kriz, J., Canzler, S., Gameiro, R. R., Krapp, V., et al. (2013). Emotions' impact on viewing behavior under natural conditions. PLoS ONE 8:e52737. doi: 10.1371/journal.pone.0052737

Kaspar, K., and König, P. (2012). Emotions and personality traits as highlevel factors in visual attention: a review. Front. Hum. Neurosci. 6:321. doi: 10.3389/fnhum.2012.00321

Knight, M., Seymour, T. L., Gaunt, J. T., Baker, C., Nesmith, K., and Mather, M. (2007). Aging and goal-directed emotional attention: distraction reverses emotional biases. Emotion 7, 705-714. doi: 10.1037/1528-3542. 7.4.705

Kollmorgen, S., Nortmann, N., Schröder, S., and König, P. (2010). Influence of lowlevel stimulus features, task dependent factors, and spatial biases on overt visual attention. PLoS Comput. Biol. 6, e1000791. doi: 10.1371/journal.pcbi.1000791

Koster, E. H., De Raedt, R., Leyman, L., and De Lissnyder, E. (2010). Mood-congruent attention and memory bias in dysphoria: exploring the coherence among information-processing biases. Behav. Res. Ther. 48, 219-225. doi: 10.1016/j.brat.2009.11.004

Krieglmeyer, R., Deutsch, R., De Houwer, J., and De Raedt, R. (2010). Being moved: valence activates approach-avoidance behavior independently of evaluation and approach-avoidance intentions. Psychol. Sci. 21, 607-613. doi: 10.1177/0956797610365131

Kuznetsova, A., Brockhoff, P. B., and Christensen, R. H. B. (2017). lmerTest package: tests in linear mixed effects models. J. Stat. Softw. 82, 1-26. doi: 10.18637/jss.v082.i13

Lang, P. J., Bradley, M. M., and Cuthbert, B. N. (1997). International Affective Picture System (IAPS): Technical Manual and Affective Ratings. NIMH Center for the Study of Emotion and Attention, 39-58.

Lavender, T., and Hommel, B. (2007). Affect and action: towards an event-coding account. Cogn. Emot. 21, 1270-1296. doi: 10.1080/02699930701438152

Marmolejo-Ramos, F., Arshamian, A., Tirado, C., Ospina, R., and Larsson, M. (2019). The allocation of valenced percepts onto 3D space. Front. Psychol. 10:352. doi: 10.3389/fpsyg.2019.00352

Marmolejo-Ramos, F., Cousineau, D., Benites, L., and Maehara, R.-o. (2015). On the efficacy of procedures to normalize Ex-Gaussian distributions. Front. Psychol. 5:1548. doi: 10.3389/fpsyg.2014.01548

Marmolejo-Ramos, F., Tirado, C., Arshamian, E., Vélez, J. I., and Arshamian, A. (2018). The allocation of valenced concepts onto 3D space. Cogn. Emot. 32, 709-718. doi: 10.1080/02699931.2017.1344121

Mather, M., and Knight, M. R. (2006). Angry faces get noticed quickly: threat detection is not impaired among older adults. J. Gerontol. Ser. B Psychol. Sci. Soc. Sci. 61, P54-P57. doi: 10.1093/geronb/61.1.P54

Niedenthal, P. M., Barsalou, L. W., Winkielman, P., Krauth-Gruber, S., and Ric, F. (2005). Embodiment in attitudes, social perception, and emotion. Pers. Soc. Psychol. Rev. 9, 184-211. doi: 10.1207/s15327957pspr0903_1

Ossandon, J. P., Onat, S., and Konig, P. (2014). Spatial biases in viewing behavior. J. Vis. 14:20. doi: 10.1167/14.2.20

Parrott, W. G., and Sabini, J. (1990). Mood and memory under natural conditions: evidence for mood incongruent recall. J. Pers. Soc. Psychol. 59:321. doi: 10.1037/0022-3514.59.2.321

Phaf, R. H., Mohr, S. E., Rotteveel, M., and Wicherts, J. M. (2014). Approach, avoidance, and affect: a meta-analysis of approach-avoidance tendencies in manual reaction time tasks. Front. Psychol. 5:378. doi: $10.3389 /$ fpsyg. 2014.00378
Rayner, K. (2009). The 35th Sir Frederick Bartlett Lecture: eye movements and attention in reading, scene perception, and visual search. Q. J. Exp. Psychol. 62, 1457-1506. doi: 10.1080/17470210902816461

Rinck, M., and Becker, E. S. (2007). Approach and avoidance in fear of spiders. J. Behav. Ther. Exp. Psychiatry 38, 105-120. doi: 10.1016/j.jbtep.2006.10.001

Rothkopf, C. A., Ballard, D. H., and Hayhoe, M. M. (2016). Task and context determine where you look. J. Vis. 7:16. doi: 10.1167/7.14.16

Rowe, G., Hirsh, J. B., and Anderson, A. K. (2007). Positive affect increases the breadth of attentional selection. Proc. Natl. Acad. Sci. U.S.A. 104, 383-388. doi: 10.1073/pnas.0605198104

Schwager, S., and Rothermund, K. (2013). Counter-regulation triggered by emotions: positive/negative affective states elicit opposite valence biases in affective processing. Cogn. Emot. 27, 839-855. doi: 10.1080/02699931.2012.750599

Schwarz, N. (1990). "Feelings as information: informational and motivational functions of affective states," in Handbook of Motivation and Cognition: Foundations of Social Behavior, Vol. 2, eds E. T. Higgins and R. M. Sorrentino (New York city, NY: The Guilford Press), 527-561.

Shapiro, K. L., and Lim, A. (1989). The impact of anxiety on visual attention to central and peripheral events. Behav. Res. Ther. 27, 345-351. doi: 10.1016/0005-7967(89)90004-1

Shapiro, L. (2011). Embodied Cognition. Routledge. doi: $10.5840 /$ philtopics201139117

Sharbanee, J. M., Hu, L., Stritzke, W. G. K., Wiers, R. W., Rinck, M., and MacLeod, C. (2014). The effect of approach/avoidance training on alcohol consumption is mediated by change in alcohol action tendency. PLoS ONE 9:e85855. doi: 10.1371/journal.pone.0085855

Slaby, J., Paskaleva, A., and Stephan, A. (2016). Enactive emotion and impaired agency in depression. J. Conscious. Stud. 20, 33-55. Availabe online at: https:// www.ingentaconnect.com/content/imp/jcs/2013/00000020/f0020007/art00003

Stephan, A., Walter, S., and Wilutzky, W. (2014). Emotions beyond brain and body. Philos. Psychol. 27, 65-81. doi: 10.1080/09515089.2013.828376

Stoykov, M. E., Corcos, D. M., and Madhavan, S. (2017). Movement-based priming: clinical applications and neural mechanisms. J. Motor Behav. 49, 88-97. doi: 10.1080/00222895.2016.1250716

Tatler, B. W. (2007). The central fixation bias in scene viewing: selecting an optimal viewing position independently of motor biases and image feature distributions. J. Vis. 7:4. doi: 10.1167/7.14.4

Thomas, R. C., and Hasher, L. (2006). The influence of emotional valence on age differences in early processing and memory. Psychol. Aging 21, 821-825. doi: 10.1037/0882-7974.21.4.821

Tomaszczyk, J. C., Fernandes, M. A., and Macleod, C. M. (2008). Personal relevance modulates the positivity bias in recall of emotional pictures in older adults. Psychon. Bull. Rev. 15, 191-196. doi: 10.3758/PBR.15.1.191

Treisman, A. M., and Gelade, G. (1980). A feature-integration theory of attention. Cogn. Psychol. 12, 97-136. doi: 10.1016/0010-0285(80)90005-5

Veenstra, E. M., and de Jong, P. J. (2011). Reduced automatic motivational orientation towards food in restricting anorexia nervosa. J. Abnorm. Psychol. 120, 708-718. doi: 10.1037/a0023926

Wadlinger, H. A., and Isaacowitz, D. M. (2006). Positive mood broadens visual attention to positive stimuli. Motiv. Emot. 30, 87-99. doi: $10.1007 / \mathrm{s} 11031-006-9021-1$

Wadlinger, H. A., and Isaacowitz, D. M. (2011). Fixing our focus: training attention to regulate emotion. Pers. Soc. Psychol. Rev. 15, 75-102. doi: $10.1177 / 1088868310365565$

Walter, S. (2014). Situated cognition: a field guide to some open conceptual and ontological issues. Rev. Philos. Psychol. 5, 241-263. doi: 10.1007/s13164-013-0167-y

Wiers, C. E., Kühn, S., Javadi, A. H., Korucuoglu, O., Wiers, R. W., Walter, H., et al. (2013). Automatic approach bias towards smoking cues is present in smokers but not in ex-smokers. Psychopharmacology 229, 187-197. doi: 10.1007/s00213-013-3098-5

Wiers, C. E., Stelzel, C., Park, S. Q., Gawron, C. K., Ludwig, V. U., Gutwinski, S., et al. (2014). Neural correlates of alcohol-approach bias in alcohol addiction: the spirit is willing but the flesh is weak for spirits. Neuropsychopharmacology 39, 688-697. doi: 10.1038/npp.2013.252

Wiers, R. W., Eberl, C., Rinck, M., Becker, E. S., and Lindenmeyer, J. (2011). Retraining automatic action tendencies changes alcoholic patients' approach 
bias for alcohol and improves treatment outcome. Psychol. Sci. 22, 490-497. doi: 10.1177/0956797611400615

Wiers, R. W., Gladwin, T. E., Hofmann, W., Salemink, E., and Ridderinkhof, K. R. (2013a). Cognitive bias modification and cognitive control training in addiction and related psychopathology: mechanisms, clinical perspectives, and ways forward. Clin. Psychol. Sci. 1, 192-212. doi: 10.1177/2167702612466547

Wiers, R. W., Gladwin, T. E., and Rinck, M. (2013b). Should we train alcohol-dependent patients to avoid alcohol? Front. Psychiatry 4:33. doi: 10.3389/fpsyt.2013.00033

Wiers, R. W., Rinck, M., Dictus, M., and van den Wildenberg, E. (2009). Relatively strong automatic appetitive action-tendencies in male carriers of the OPRM1 G-allele. Genes Brain Behav. 8, 101-106. doi: 10.1111/j.1601-183X.2008.00454.x

Wilming, N., Harst, S., Schmidt, N., and König, P. (2013). Saccadic momentum and facilitation of return saccades contribute to an optimal foraging strategy. PLoS Comput. Biol. 9:e1002871. doi: 10.1371/journal.pcbi.1002871

Witt, J. K., and Proffitt, D. R. (2008). Action-specific influences on distance perception: a role for motor simulation. J. Exp. Psychol. 34, 1479-1492. doi: 10.1037/a0010781
Xing, C., and Isaacowitz, D. M. (2006). Aiming at happiness: how motivation affects attention to and memory for emotional images. Motiv. Emot. 30, 243-250. doi: 10.1007/s11031-006-9032-y

Zhou, Y., Li, X., Zhang, M., Zhang, F., Zhu, C., and Shen, M. (2012). Behavioural approach tendencies to heroin-related stimuli in abstinent heroin abusers. Psychopharmacology 221, 171-176. doi: 10.1007/s00213-011-2557-0

Conflict of Interest: The authors declare that the research was conducted in the absence of any commercial or financial relationships that could be construed as a potential conflict of interest.

Copyright (c) 2021 Czeszumski, Albers, Walter and König. This is an open-access article distributed under the terms of the Creative Commons Attribution License (CC $B Y)$. The use, distribution or reproduction in other forums is permitted, provided the original author(s) and the copyright owner(s) are credited and that the original publication in this journal is cited, in accordance with accepted academic practice. No use, distribution or reproduction is permitted which does not comply with these terms. 